\title{
Low Noise High-Energy Dissipative Soliton Erbium Fiber Laser for Fiber Optical Parametric Oscillator Pumping
}

\author{
Mincheng Tang ${ }^{1}$, Rezki Becheker ${ }^{1,2}{ }^{\circledR}$, Pierre-Henry Hanzard ${ }^{1}$, Aleksey Tyazhev ${ }^{1}$, \\ Jean-Louis Oudar ${ }^{3}$, Arnaud Mussot ${ }^{4}$, Alexandre Kudlinski ${ }^{4}$, Thomas Godin ${ }^{1, *}$ (I) and \\ Ammar Hideur ${ }^{1}$ \\ 1 CORIA UMR 6614, Normandie Université, CNRS-INSA-Université de Rouen Normandie, F-76800 \\ Saint-Etienne du Rouvray, France; mincheng.tang@coria.fr (M.T.); becheker@coria.fr (R.B.); \\ hanzardp@coria.fr (P.-H.H.); tyazheva@coria.fr (A.T.); hideur@coria.fr (A.H.) \\ 2 Laboratoire d'Electronique Quantique, USTHB, BP 32 El Alia, 16111 Bab Ezzouar, Algeria \\ 3 Centre for Nanoscience and Nanotechnology, CNRS, Université Paris-Sud, Université Paris-Saclay, \\ F-91460 Marcoussis, France; jean-louis.oudar@c2n.upsaclay.fr \\ 4 CNRS, UMR 8523-PhLAM, Université de Lille 1, F-59000 Lille, France; \\ Arnaud.Mussot@phlam.univ-lille1.fr (A.M.); alexandre.kudlinski@univ-lille1.fr (A.K.) \\ * Correspondence: thomas.godin@coria.fr; Tel.: +33-232-95-37-38
}

Received: 27 September 2018; Accepted: 30 October 2018; Published: 5 November 2018

\begin{abstract}
We report on a mode-locked erbium-doped fiber laser delivering highly-chirped pulses with several tens of nanojoules of energy around $1560 \mathrm{~nm}$ and its exploitation to efficiently pump a fiber optical parametric oscillator (FOPO), thus enabling picosecond pulse generation around $1700 \mathrm{~nm}$. The laser cavity features a high normal dispersion and mode-locking is sustained using tailored spectral filtering combined with nonlinear polarization evolution and a semiconductor saturable absorber. Numerical simulations show that the laser dynamics is governed by a strong mode-locking mechanism compensating for the large spectral and temporal pulse evolution along the cavity. In the frame of high energy picosecond pulse generation around $1700 \mathrm{~nm}$, we then demonstrate that using highly-chirped pulses as pump pulses allows for the efficient tuning of the FOPO idler wavelength between 1620 and $1870 \mathrm{~nm}$. In addition, satisfying noise characteristics have been achieved both for the Er-laser and the FOPO, with respective relative intensity noises (RIN) of -154 and $-140 \mathrm{dBc} / \mathrm{Hz}$, thus paving the way for the use of such sources in ultrafast instrumentation.
\end{abstract}

Keywords: erbium fiber laser; mode-locked lasers; parametric oscillators; dissipative solitons

\section{Introduction}

The development of high-energy ultrafast fiber lasers has attracted much interest owing to their inherent advantages including compactness, high stability, high-power capability, and turnkey operation. Motivated by demands from several key industrial and scientific applications, such as material processing, nonlinear microscopy, and high-field physics, impressive progress has been achieved in the past few decades due to both the development of novel fiber technologies and the discovery and control of novel mode-locking regimes, especially the exploitation of dissipative soliton regimes, in which significant energy scaling is achieved [1-4]. Erbium-doped fiber lasers are now a widespread technology in many applications such as free-space telecommunications, range finding, remote sensing, and medical procedures. Another category of applications of interest concerns the generation of ultrashort pulses outside the wavelength window covered by rare-earth-based fiber lasers, in particular for applications in biophotonics [5,6] and sensing [7], where low-noise pulses 
are required. In this frame, efficient fiber laser systems based on frequency conversion processes, e.g., four-wave mixing (FWM), have been developed, enabling wavelength tunability over several hundreds of nanometers [8]. To date, the best performances in terms of noise are obtained with synchronously pumped fiber optical parametric oscillators (FOPOs). Their combination with spectral or dispersion-based filters allowed broad tunability and high energies in the $0.7-1.3 \mu \mathrm{m}$ wavelength range $[5,6,9]$. The extension of this technology in the range around $1.7 \mu \mathrm{m}$, which is attractive due to the enhanced penetration depth in nonlinear bio-imaging [10], and is now a challenge attracting strong attention from the scientific community [11-17]. Different fiber-based solutions have then been proposed, from systems relying on soliton self-frequency shift $[10,18,19]$ to a FOPO driven by a master-oscillator-power-amplifier (MOPA) [12,20,21], to name a few. The noise characteristics of these systems and/or the use of bulky and fragile components, such as rod-type fibers, are however limiting factors for real-world applications in nonlinear imaging or ultrafast instrumentation. There is thus an identified need not only for FOPO systems emitting around $1.7 \mu \mathrm{m}$ but also for efficient and versatile fiberized pump sources featuring at the same time relatively high energies, good noise characteristics, and the possibility to directly generate highly-chirped pulses as, in this case, dispersive tuning within the FOPO cavity can offer a supplementary degree of freedom for the FOPO's tunability.

A common method developed to initially obtain high-energy ultrashort pulses from Er-doped fiber lasers relies on the principle of a chirped pulse oscillator (CPO), which takes advantage of the concept of a dissipative soliton laser [22-24]. In this configuration, stable mode-locked operation can be achieved using strong mode-locking mechanisms, which could include either components with high-nonlinearity such as semiconductor saturable absorber mirrors (SESAMs) [25] or nonlinear polarization evolution (NPE). The pulses are then highly chirped due to the high-normal group dispersion delay (GDD) nature of such oscillators and could be linearly de-chirped to produce ultrashort pulses with high peak powers.

In this article, we first review the various developments of the all-normal-dispersion fiber lasers in the last few years. We then report and review the design and realization of a large normal dispersion mode-locked erbium-doped fiber laser emitting dissipative solitons at $1560 \mathrm{~nm}$ and use numerical simulations to highlight the specific self-similar dynamics occurring within the cavity. We then show that this source possesses the particular above-mentioned characteristics in terms of noise and energy to be efficiently used as the pumping source of a FOPO in order to generate picosecond pulses around $1.7 \mu \mathrm{m}$. We also evaluate the noise performances of both the pump and parametric pulses in order to attest to the possibility of using such an ultrafast system for specific applications such as nonlinear imaging.

\section{Materials and Methods}

\subsection{Large Normal Dispersion Er-Fiber Laser: State-of-the-Art and Our Contribution}

In 2006, Andy et al. experimentally demonstrated for the first time the possibility and potential of normal dispersion regimes regarding the energy scaling [26]. They reported the generation of $3 \mathrm{~nJ}$ pulses that could be de-chirped to $170 \mathrm{fs}$ from an "All Normal Dispersion" (ANDi) fiber laser based on an ytterbium-doped active fiber without any dispersive delay line or anomalous dispersion in the cavity. Further exploitation of this idea significantly increased the performances with an optimized design leading to the production of 200-fs pulses reaching the energy of $20 \mathrm{~nJ}$ from an ytterbium-doped fiber oscillator operating at $1 \mu \mathrm{m}$ wavelength [23]. This energy scaling was possible because the effect of nonlinear phase accumulation in such a regime could be compensated by a strong pulse-shaping mechanism provided by a passive spectral filter, which is also a key mechanism for the laser operations presented in this review and will be addressed later. It is worth noting that the combination of this concept with large mode-area ytterbium doped fibers has allowed to reach record pulse energies directly produced from a fiber oscillator by approaching the $\mu \mathrm{J}$ level [2-4]. 
To generalize this method to fiber lasers emitting at $\approx 1550 \mathrm{~nm}$, the main limitation lies in the fact that commercially available components are mainly based on normal single mode fibers, such as SMF28, OFS980, or Hi1080, which exhibit anomalous dispersion at this wavelength. In 2010, Nikolai et al. reported high-power dissipative soliton generation from an all-normal dispersion erbium fiber oscillator [27]. The Metrocor fiber from Corning, which has an anomalous dispersion of $9.5 \mathrm{ps}^{2} / \mathrm{km}$, was used to build the laser cavity and the key component enabling the all-normal-dispersion design was a homemade wavelength division multiplexer (WDM) based on the Metrocor fiber. With this laser setup, they succeeded in generating 53-ps pulses with energy of $20 \mathrm{~nJ}$, and the pulses could be de-chirped to $750 \mathrm{fs}$. The de-chirped pulse quality was however compromised as it exhibits a large pedestal as revealed by the auto-correlation measurements. This phenomenon is attributed to the uncompensated third-order dispersion, which is estimated to be around $0.2 \mathrm{ps}^{3}$ by simulation matching the auto-correlation (AC) trace. Later, in 2014, a self-similar Er-fiber laser drawing on this concept was demonstrated [28]. It produced high-energy ( $3.5 \mathrm{~nJ})$ chirped pulses with a spectrum spreading from $1500 \mathrm{~nm}$ to $1570 \mathrm{~nm}$, which could be de-chirped down to $70 \mathrm{fs}$. Most of these results have been achieved using nonlinear polarization evolution as a mode-locking mechanism in combination with a passive spectral filter. In this section, we discuss the features of a dissipative soliton laser operation at large normal dispersion and using a high nonlinearity semiconductor saturable absorber in combination with a nonlinear polarization.

The typical design of our large normal dispersion oscillator, based on the principle of $\mathrm{CPO}$, is schematically described in Figure 1 and in Ref. [29]. The gain medium is a piece of $1.2 \mathrm{~m}$ heavily erbium-doped single mode fiber (EDF), which has a mode field diameter of $4.3 \mu \mathrm{m}$ and a numerical aperture of 0.29 at $\approx 1550 \mathrm{~nm}$. The EDF presents an unpumped peak absorption of $80 \mathrm{~dB} / \mathrm{m}$ at $1530 \mathrm{~nm}$, and still exhibits normal group velocity dispersion (GVD) around $1550 \mathrm{~nm}$. The dispersion compensating fiber (DCF) is the main component used to introduce and optimize high normal dispersion in the oscillator. A single-mode Yb-doped fiber laser (Azur light system Inc., Pessac, France), which can supply up to $5 \mathrm{~W}$ optical power output, was used to pump the system through a $980 / 1550$ WDM. A 90/10 coupler was used to extract $90 \%$ of the power out of the cavity. One end of the Fabry-Pérot cavity consisted in a resonant saturable absorber mirror (R-SAM, developed by C2N laboratory, Marcoussis, France), whose modulation depth was $35 \%$ and relaxation time was 2 ps, along with coupling lenses and a polarization controller, providing nonlinear reflectivity acting as the mode-locking mechanism. A blazed grating $(600$ lines $/ \mathrm{mm})$ was used at the other end of the cavity with a coupling lens, which together formed a Gaussian profile bandpass filter allowing us to stabilize the laser oscillation, adjust the operation wavelength, and ensure pulse self-consistency.

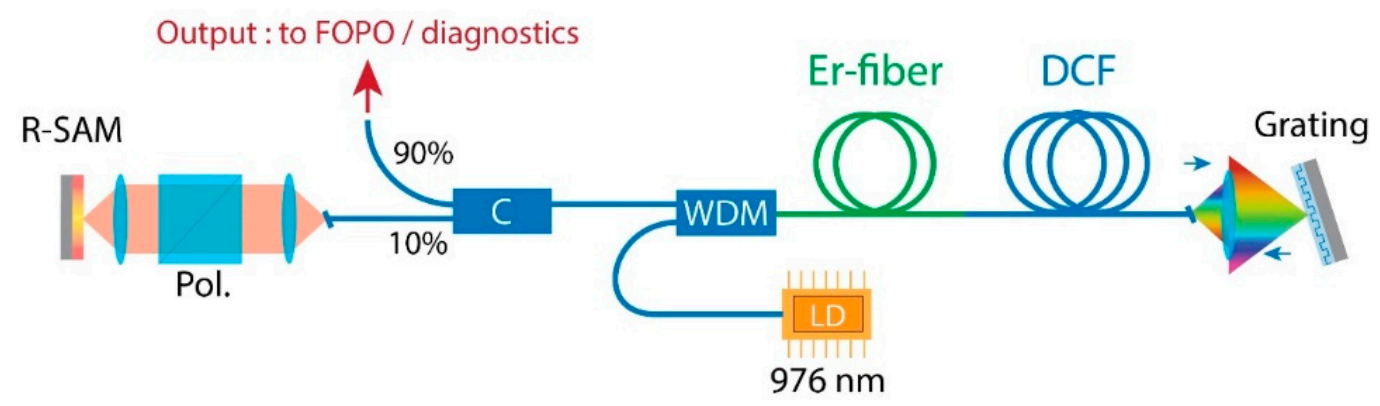

Figure 1. Er-fiber laser experimental setup. C: coupler; WDM: wavelength division multiplexer; DCF: dispersion compensating fiber; LD: laser diode.

Compared to the previous works mentioned above, which all take the design of a ring cavity to use the NPE as the mode-locking mechanism, we based our set-up on the Fabry-Pérot cavity design with the SESAM incorporation, which has proved practical in the previous work of our group reported in 2011 [25], where different SESAMs were tested for a laser with a similar but more primitive design. 


\subsection{Numerical Simulations}

In order to characterize and understand the pulse dynamics inside this cavity and have a prospect of the output performances, we performed numerical simulations using commercial software (Fiberdesk, Ref. [30]) allowing us to resolve the extended nonlinear Schrödinger equation (NLSE):

$$
\frac{\partial A}{\partial z}=g(\mathrm{E}) A+\frac{i g}{\Delta \Omega_{g}^{2}} \frac{\partial^{2} A}{\partial t^{2}}+\sum_{m \geq 2} \frac{i^{m+1}}{m !} \beta_{m} \frac{\partial^{m} A}{\partial t^{m}}+i \gamma|A|^{2} A,
$$

where $A$ is the slowly varying complex pulse envelope, $\beta_{m}$ is the $m$ th order dispersion coefficient, the $i g / \Delta \Omega_{g}^{2}$ term denotes spectral filtering due to the limited gain bandwidth, and $\gamma$ corresponds to the contribution from Kerr non-linearity through self-phase modulation and is classically defined as:

$$
\gamma=\frac{n_{2} \omega_{0}}{c A_{\text {eff }}}
$$

where $n_{2}$ is the nonlinear index and $A_{\text {eff }}$ is the effective mode field area. The effect of gain saturation is indicated by $g$, which stands for the saturated gain that is related to low signal gain $g_{0}$ by:

$$
g(E)=g_{0} /\left(1+\frac{E}{E_{s a t}}\right)
$$

where $E_{\text {sat }}$ is the saturation energy transferable from the pump.

Equation (1) is solved using the split-step method along the cavity elements, as depicted in Figure 2 and with the parameters listed in Table 1. The effect of the real R-SAM and of the polarization controller are modeled as a single effective SAM with $90 \%$ modulation depth, which is a modulation depth that is fairly rational for the nonlinear effect of R-SAM and the NPE combined.

Table 1. Fiber parameters used in the simulation.

\begin{tabular}{ccccc}
\hline Parameter & Units & Er-Fiber & SMF & DCF \\
\hline Length & $\mathrm{m}$ & 1.2 & 0.6 & 10 \\
Mode field diameter & $\mu \mathrm{m}$ & 4.9 & 10 & 6 \\
GVD $\left(\beta_{2} @ 1550 \mathrm{~nm}\right)$ & $\mathrm{ps}^{2} / \mathrm{m}$ & +0.06 & -0.023 & +0.116 \\
Effective area $\left(A_{\text {eff }}\right)$ & $\mu \mathrm{m}^{2}$ & 18.8 & 78.5 & 28.26 \\
Nonlinear coefficient $(\gamma)$ & $\mathrm{W}^{-1} \cdot \mathrm{m}^{-1}$ & $6.4 \times 10^{-3}$ & $1.5 \times 10^{-3}$ & $4.2 \times 10^{-3}$ \\
Peak small signal gain $(g 0)$ & $\mathrm{m}^{-1}$ & 4.6 & 0 & 0 \\
Gain bandwidth $\left(\Delta \lambda_{g}\right)$ & $\mathrm{nm}$ & 10 & - & - \\
\hline
\end{tabular}

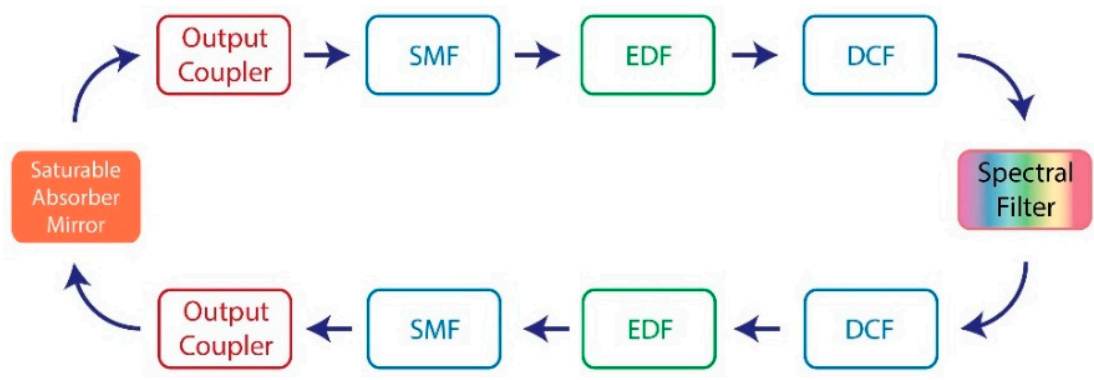

Figure 2. Schematic representation of the unfolded cavity, as used in the numerical simulations. SMF: single-mode fiber.

\section{Numerical and Experimental Results}

We first look at the manner in which characteristic parameters of the output pulses under mode-locking operations would evolve with the increase of pump level. The results are shown 
in Figure 3, where the pump power is represented by the gain saturation energy of the doped fiber. Pump powers beyond the limit shown in the figures are also emulated, and the results are not included because only undesired multi-pulse operations are retrievable.

(a)

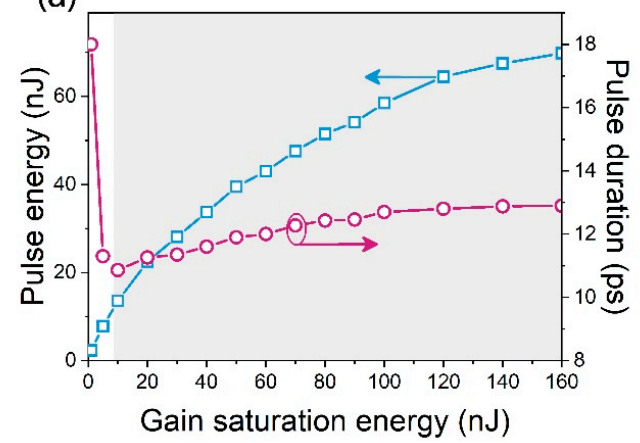

(b)

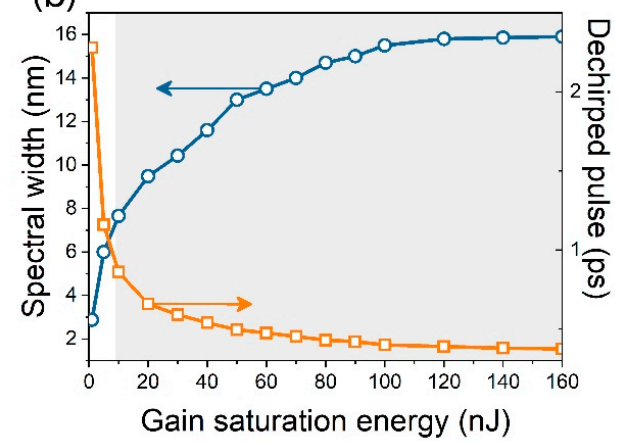

Figure 3. Simulated evolution of pulse parameters with respect to pump power. Shaded areas represent energy levels for which single and multiple pulse regimes coexist.

We can see that the pulse energy, duration, and spectral bandwidth monotonically increased while the duration of the de-chirped pulse monotonically decreased. The simulation predicted pulse energies up to $70 \mathrm{~nJ}$ for this cavity design, and with a spectral bandwidth of $\approx 16 \mathrm{~nm}$, the de-chirped pulse duration could reach less than $400 \mathrm{fs}$. It should be noticed that at higher energy levels, single and multiple pulse operations coexist, meaning that mode transition is possible in our configuration, which uses an NPE-SAM cooperated mode-locking mechanism. We then characterized the pulse properties under different GDD configuration (i.e., for different lengths of DCF) so that the impact of the dispersion management to the energy scaling could be compared and studied. As we focused on high-energy pulse delivery, the results and discussion concerning the laser with similar configuration but with very low normal GDD are not discussed and can be found in Ref. [31].

Motivated by the results of the simulations, we first built the oscillator with $10 \mathrm{~m}$ of DCF inside the cavity, corresponding to a cavity length of $\approx 12.5 \mathrm{~m}$ and a GDD around $+1.22 \mathrm{ps}^{2}$. Stable mode-locking was achievable by adjusting the focusing conditions of the coupling lenses and fine-tuning of the polarization components. Figure 4 shows the output pulse characterizations of a typical regime under this configuration: the pulse energy was about $22 \mathrm{~nJ}$ derived from the measured average power of $176 \mathrm{~mW}$ at a repetition rate of $7.9 \mathrm{MHz}$. The pulse duration was $12.4 \mathrm{ps}$ (NB: measured pulse durations were inferred from the AC trace full width at half maximum (FWHM), and the same criterion applies to all following measurements unless otherwise mentioned. The values are $\sqrt{2}$ times the actual pulses' durations.) and could be de-chirped down to $940 \mathrm{fs}$ outside the cavity using a pair of transmission gratings with 1000 lines $/ \mathrm{mm}$ and $92 \%$ diffraction efficiency (Figure $4 \mathrm{~b}$ ), which is very close to the Fourier limit of its $8.1 \mathrm{~nm}$ bandwidth spectrum. The required de-chirping dispersion was measured to be about $-0.416 \mathrm{ps}^{2}$. We also observe the transitions between single-pulse and multi-pulse operations when the pump power was increased, a phenomenon predicted by the simulation, which was mitigated by careful tuning of the polarization components; this is an indication of NPE being a part of the mode-locking mechanism.

We then increased the DCF length to $15 \mathrm{~m}$, consequently increasing the cavity GDD to $+1.83 \mathrm{ps}^{2}$, its length to $\approx 20 \mathrm{~m}$, and reducing the repetition rate to $5.33 \mathrm{MHz}$ for the single pulse mode-locked operations. Stable mode-locking regimes were achievable with the same tuning methods as in the previous stage and Figure 5 shows the typical output pulse characteristics of such regimes. An average optical power of $204 \mathrm{~mW}$ was measured from the laser output, corresponding to $38 \mathrm{~nJ}$ of pulse energy. The pulse duration was $14.5 \mathrm{ps}$, and could be compressed to $1 \mathrm{ps}$ with the same de-chirping mechanism. The de-chirped pulse's AC is shown in Figure 5b, together with the Fourier limit calculated from the measured spectrum (shown in Figure 5a), from which it can be concluded that the pulse was 
de-chirped to 1.2 times its Fourier limit at best. This configuration brought a higher pulse energy and a shorter pulse duration, but with decreased pulse quality, as the pulse could not be de-chirped completely. This was attributed to excessive nonlinear phase in the pulse indicating that the linear stretching effect of dispersion was not enough to compensate for the nonlinearity introduced by high peak powers. The results of configurations with longer DCF lengths in the cavity confirming this method could not be extended to achieve a much better energy scaling.

(a)

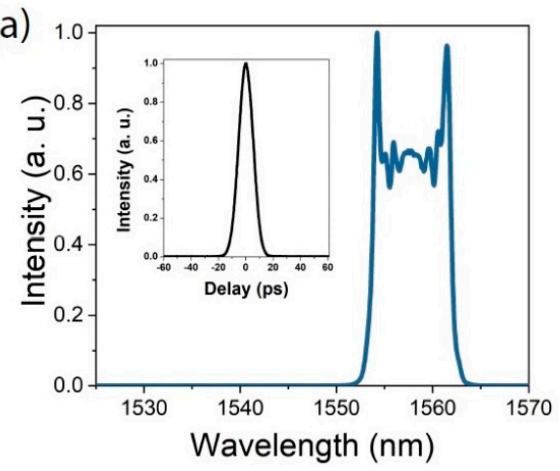

(b)

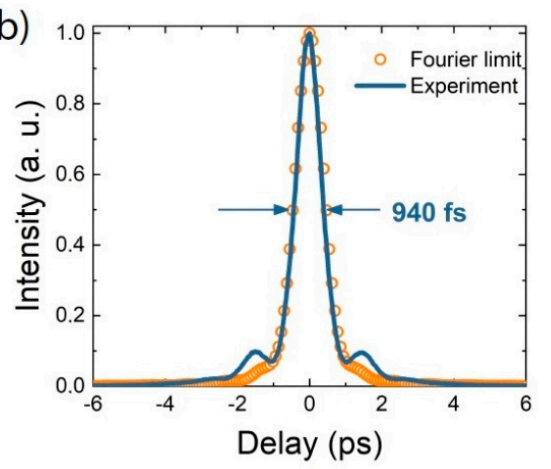

Figure 4. Pulse characterization of configuration with a 10-m DCF in the cavity. (a) Spectrum (inset: AC trace). (b) AC trace of the de-chirped pulse and calculated Fourier limit of the pulse.

(a)

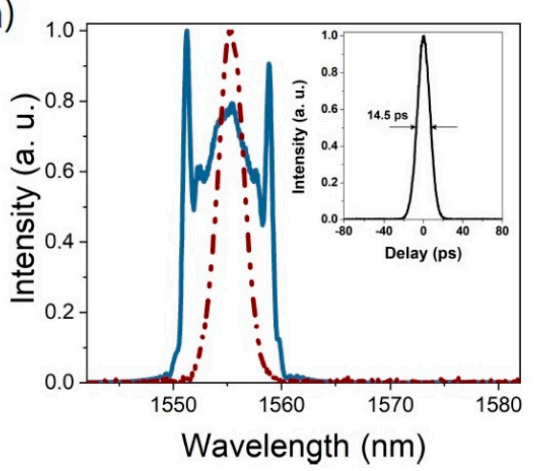

(b)

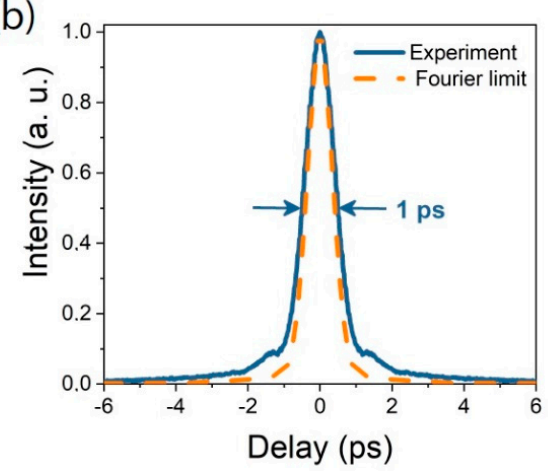

Figure 5. Characterization of a mode-locking regime with 15-m-DCF-in-cavity configuration. (a) spectrum and AC trace (inset), where the brown dashed line indicates the filtering effect of the grating-lens combination. (b) AC trace of the de-chirped pulse and its comparison to the Fourier limit.

We eventually implemented configurations with higher GDD, for instance using $30 \mathrm{~m}$ of DCF in the cavity, which corresponded to a GDD of $+3.57 \mathrm{ps}^{2}$. In this case, the stability of the mode-locking regimes was more and more compromised in this process as Q-switching mode-locking regimes started to dominate the laser operation.

\section{Discussion and Application}

\subsection{Intracavity Dynamics}

Simulations were carried out to better understand the intracavity evolution of the pulse. The filtering effect was measured to be equivalent to a $3.6 \mathrm{~nm}$ FWHM Gaussian profile bandpass filter by splicing a $2 \times 2-10 / 90$ coupler to the DCF and comparing the measured spectra from both $10 \%$ output ports. The coupler was minimized in length and has negligible influence on GDD, which was confirmed by the unchanged pulse parameters measured after its incorporation. With a 10-m long DCF, the numerical simulations were in good agreement with the experimental results shown in Figure 4, as the main pulse features were reproduced. Figure 6 indeed shows the simulated pulse spectrum and temporal profile at a gain saturation energy of $15 \mathrm{~nJ}$, corresponding to a pulse energy of $20 \mathrm{~nJ}$. 
(a)

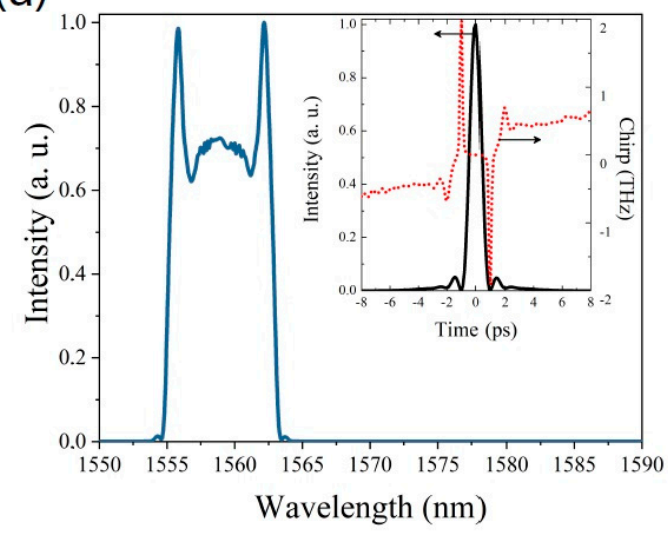

(b)

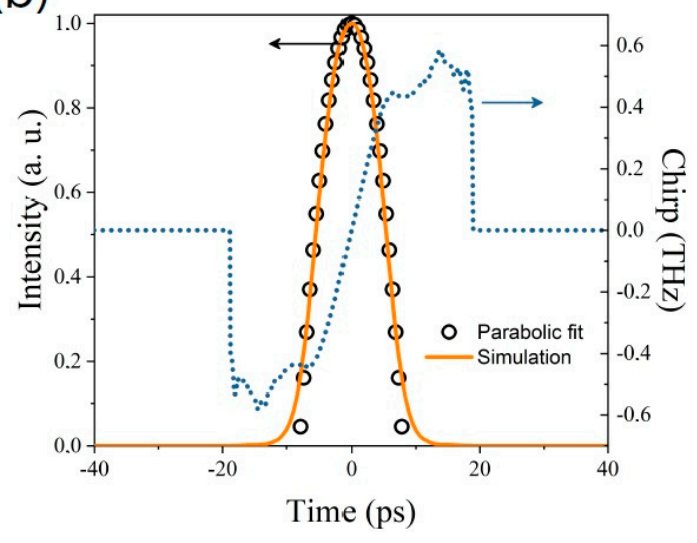

Figure 6. Simulated pulse characteristics from a cavity with a 10-m DCF and spectral filtering of $4.5 \mathrm{~nm}$.

(a) Spectral of the output pulse (inset: de-chirped pulse). (b) Corresponding temporal profile.

The simulated pulse had a spectral bandwidth of $7.7 \mathrm{~nm}$ and a quasi-parabolic temporal shape with a FWHM of $10.8 \mathrm{ps}$, and could be de-chirped to $862 \mathrm{fs}$. The fact that our simulation was in good agreement with the experimental results proved the numerical modeling of this cavity under this energy level was viable and can be used to have a better comprehension of the pulse evolution inside the cavity.

Figure 7 depicts the pulse's temporal and spectral evolution along the cavity under stable mode-locking operation. We can notice that the DCF contributes the most to the pulse stretching as expected, but also contributed the most to the SPM-induced spectral broadening, outweighing the effect from the EDF. This meant that the DCF was the main source of nonlinearity in the cavity, and explained why increasing its length to $15 \mathrm{~m}$ resulted in a regime where pulses suffered excessive nonlinear phase and could not be linearly de-chirped. It is also worth noticing that the spectral filter (SF) played an important role in the mode-locking by ensuring the pulse self-consistency. While the effect of the R-SAM was negligible at steady state, it was crucial for the mode-locking initiation.

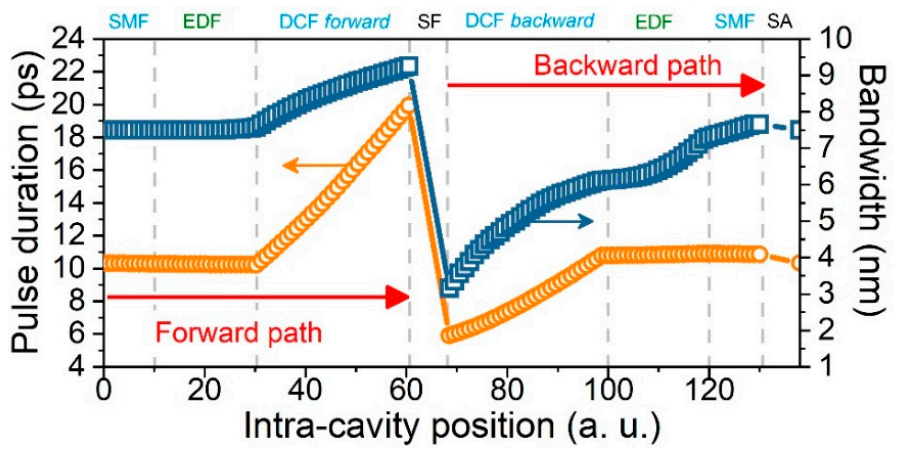

Figure 7. Pulse duration and bandwidth evolutions along the cavity elements.

The pulse shown in Figure $6 \mathrm{~b}$ was fitted assuming a parabolic shape as the common Gaussian shape was not matching. This made it worth investigating the pulse shape evolution inside the cavity. We could represent such an evolution in terms of pulse kurtosis (denoted by " $k$ "). The kurtosis is defined as $\kappa=\mu_{4} / \sigma^{4}-3$, where $\mu_{4}$ is the fourth centered moment and $\sigma^{4}$ is the square of the variance. For instance, $k=-0.86$ corresponds to a parabolic pulse shape and $k=0$ to a Gaussian shape. This metric has thereby been used to analyze laser pulse temporal shapes [30,32]. Figure 8 shows the evolution of $k$ and highlights the fact that the pulse shape evolves from a Gaussian to a parabola when propagating along the cavity. The parabolic shape was reached at the end of the DCF in the forward direction, just before the spectral filter. It was also clear that the pulse shape did not evolve significantly in the EDF and SMF, which exhibited anomalous dispersion. 


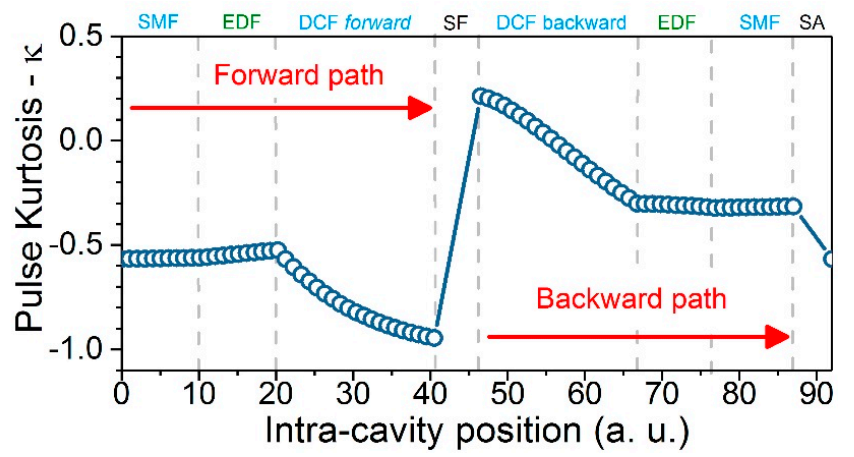

Figure 8. Evolution of the pulse shape along the cavity through its kurtosis parameter.

The performances in terms of output energy were, however, satisfying for the objective of pumping a fiber OPO based on a small-core dispersion-shifted fiber, with the extra advantage of this laser directly generating highly-chirped pulses, then enabling broad OPO tunability, as detailed in the following section.

\subsection{Application: Fiber OPO Pumping}

In the frame of achieving efficient pulse generation in the so-called biological window around $1.7 \mu \mathrm{m}$, we then used the homemade erbium fiber laser described above as the pump source of a fiber optical parametric oscillator (FOPO), as shown in Figure 9. In the configuration yielding optimal FOPO performances, the laser delivered pump pulses with a 35-ps duration, 4-nm bandwidth, and $13 \mathrm{~nJ}$, corresponding to a peak power of $370 \mathrm{~W}$. To reach such long pulse durations, the filter bandwidth was increased to lower its impact on pulse shortening inside the laser cavity. The resultant mode-locking regime exhibited longer pulses with a narrower spectra. Considering the anomalous dispersion FOPO reported here (for the idler wave), we stress that the pump pulse chirp must be high enough to allow for an overlap between the pump pulse and the idler pulse, which was long enough to get high conversion efficiencies. The chirp parameter was hence optimized by increasing the width of the pump laser intra-cavity filter, yielding pulses $\approx 40$ times longer than their Fourier limit. A fibered isolator was used to avoid any back-reflection, which could perturb the mode-locked operation. The FOPO was then pumped through the $90 \%$ port of the input coupler (C1). In its cavity, a 7-m-long dispersion shifted fiber (DSF) was used as the parametric gain medium. It had a core diameter of $3.98 \mu \mathrm{m}$, the nonlinear coefficient at $1560 \mathrm{~nm}$ was estimated to be $\gamma=6.6 \mathrm{~W}^{-1} \cdot \mathrm{km}^{-1}$, and its zero dispersion wavelength was estimated to be $1563 \pm 5 \mathrm{~nm}$. More details on the DSF can be found in Ref. [13].

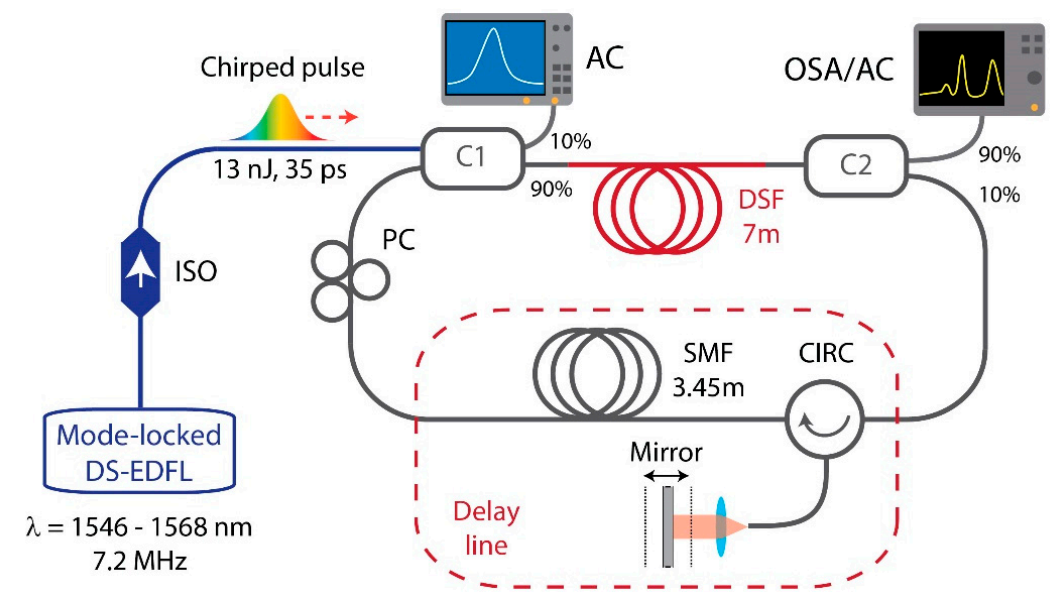

Figure 9. Experimental setup of the optical parametric oscillator [14]. 
After the DSF, $90 \%$ of the power was coupled out to diagnostics instruments through the second coupler and $10 \%$ was sent into the FOPO feedback loop. Synchronization of the resonating pulses' roundtrip time with the repetition rate of the mode-locked laser was achieved by adding an optical delay line (ODL) comprising a segment of single-mode fiber (SMF), a fiber circulator (CIRC), and free-space elements for fine adjustments. The total length of SMF (including the fiber components) used in the ODL was about $19 \mathrm{~m}$. The cavity-averaged dispersion was then estimated to $-0.095 \mathrm{ps} / \mathrm{nm}$ around $1400 \mathrm{~nm}$, and its losses were estimated to be about $29.5 \mathrm{~dB}$.

Tuning of the FOPO output wavelength could then be achieved by adjusting the two following parameters: (i) by changing the pump laser wavelength through the adjustment of its intracavity spectral filter while maintaining the same level of output energy; and (ii) via the time-dispersion-tuning technique by adjusting the FOPO cavity length, i.e., the delay in the ODL. It is worth noting that in our specific case, using highly-chirped pump pulses synchronized with the resonating idler waves at each round-trip, which are much shorter in time, provided an extra degree of freedom in wavelength selectivity as only overlapping spectral components were amplified. The combination of these two techniques allowed us to obtain a tunability of the parametric sidebands signal and idler waves ranging from 1319 to $1518 \mathrm{~nm}$, and from 1617 to $1876 \mathrm{~nm}$, respectively, as shown in Figure 10. A good agreement between experimental measurements of the FOPO sideband positions and the numerical computation of the phase-matched parametric sidebands as a function of the pump wavelength was obtained as also shown Figure 10. It is worth noting that the nanojoule level was only reached in an $80 \mathrm{~nm}$-bandwidth with wavelengths between $1640 \mathrm{~nm}$ and $1720 \mathrm{~nm}$ while pumping between $1556 \mathrm{~nm}$ and $1568 \mathrm{~nm}$.

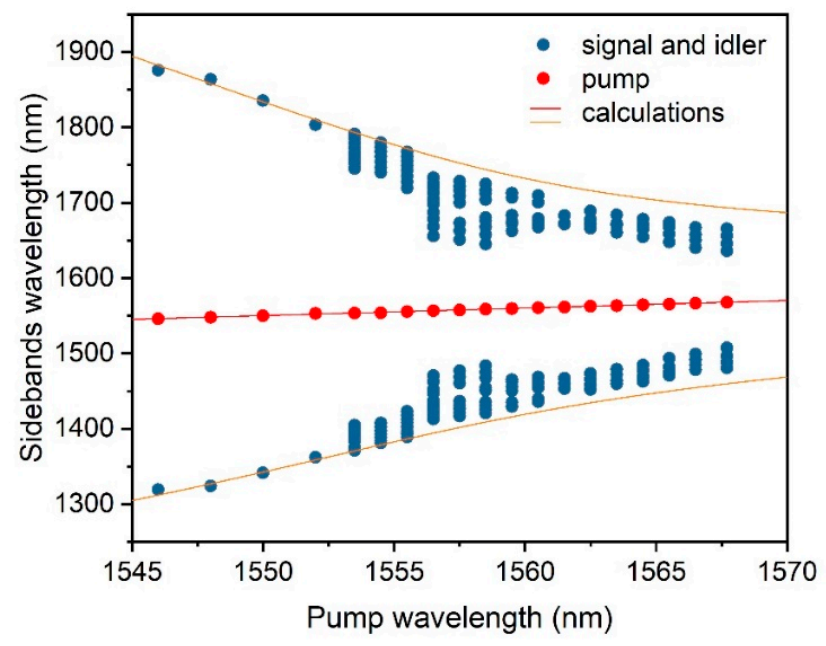

Figure 10. Experimental (blue dots) and calculated (solid lines) parametric sidebands as a function of pump wavelength (red dots).

A typical FOPO output spectrum for a pump wavelength of $1566 \mathrm{~nm}$ is then shown in Figure 11. In this particular and optimal situation, the pump was synchronized with the idler wave and the position of the delay line allowed us to obtain signal and idler waves at wavelengths of $1475 \mathrm{~nm}$ and $1668 \mathrm{~nm}$, respectively. The idler pulse duration, measured with an autocorrelator (Femtochrome FR-103XL), was found to be 2.5 times shorter than the pump pulse around 14 ps and its peak power was as high as $140 \mathrm{~W}$. The peak emission at $1530 \mathrm{~nm}$ represents the amplified spontaneous emission (ASE) in erbium, and the emission around $1600 \mathrm{~nm}$ represents the wave resulting from the interaction by FWM of the pump with the ASE at $1530 \mathrm{~nm}$. 


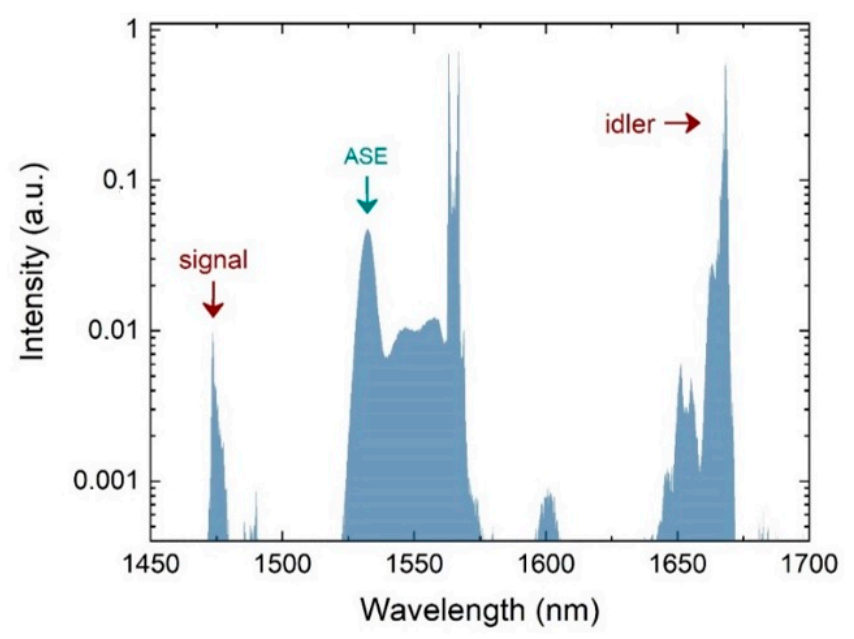

Figure 11. Typical FOPO output spectrum for a pump wavelength of $1566 \mathrm{~nm}$ with a pump-to-idler conversion efficiency of $\approx 20 \%$. The low-intensity peak around $1530 \mathrm{~nm}$ is attributed to erbium amplified spontaneous emission (ASE).

When the pump pulse at $1566 \mathrm{~nm}$ and the idler pulse were overlapped, the adjustment of the optical delay line in a range of $7 \mathrm{~mm}$ allowed for a fine tuning of the idler wave between $1620 \mathrm{~nm}$ and $1690 \mathrm{~nm}$, as can be seen from Figure 12. For this pumping wavelength, the nanojoule level was reached in a $30 \mathrm{~nm}$-bandwidth with wavelengths between $1655 \mathrm{~nm}$ and $1685 \mathrm{~nm}$. In addition, we show in Figure 13 the FOPO tuning as a function of both the pump wavelength and ODL adjustment. With these combined techniques, the idler wavelength could be tuned between $1619 \mathrm{~nm}$ and $1790 \mathrm{~nm}$ (Figure 13) when pump pulses and idler pulses were synchronized. This limitation was caused by the rapid walk-off between pump and idler pulses. Another important process that participates in the enhancement of the idler energy around this window close to $1660 \mathrm{~nm}$ was the overlapping of the Raman and FWM gain bandwidths. This Raman assistance to the parametric process can be seen as an energy transfer from the up-shifted parametric component (i.e., signal) to the down-shifted component (i.e., idler) [33]. Furthest idler wavelengths beyond $1800 \mathrm{~nm}$ could be obtained with the synchronization of the pump and signal pulses inside the FOPO cavity while pumping the DSF in its normal dispersion. We thus show the FOPO spectra for the two pump wavelengths $1546 \mathrm{~nm}$ and $1548 \mathrm{~nm}$ in Figure 14. The conversion efficiencies were relatively low compared to those in the band around $1700 \mathrm{~nm}$. When the pump was set at $1548 \mathrm{~nm}$, the signal and idler were generated at wavelengths of $1322 \mathrm{~nm}$ and $1864 \mathrm{~nm}$ with conversion efficiencies of $5.93 \%$ and $2.46 \%$, corresponding to energies of $0.23 \mathrm{~nJ}$ and $0.1 \mathrm{~nJ}$, respectively. The extreme experimental signal and idler sidebands were centered at wavelengths of $1319 \mathrm{~nm}$ and $1876 \mathrm{~nm}$ with lower conversion efficiencies of $1.15 \%$ and $0.6 \%$, respectively, for a pump wavelength of $1546 \mathrm{~nm}$ (Figure 14). 


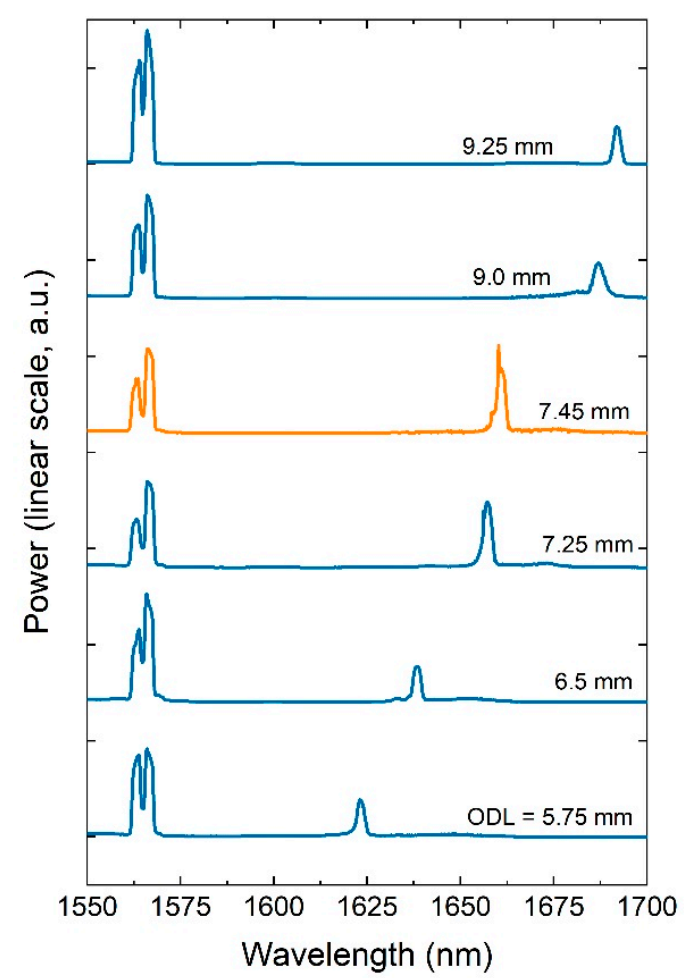

Figure 12. Idler tenability, in linear scale, while tuning the optical delay line.

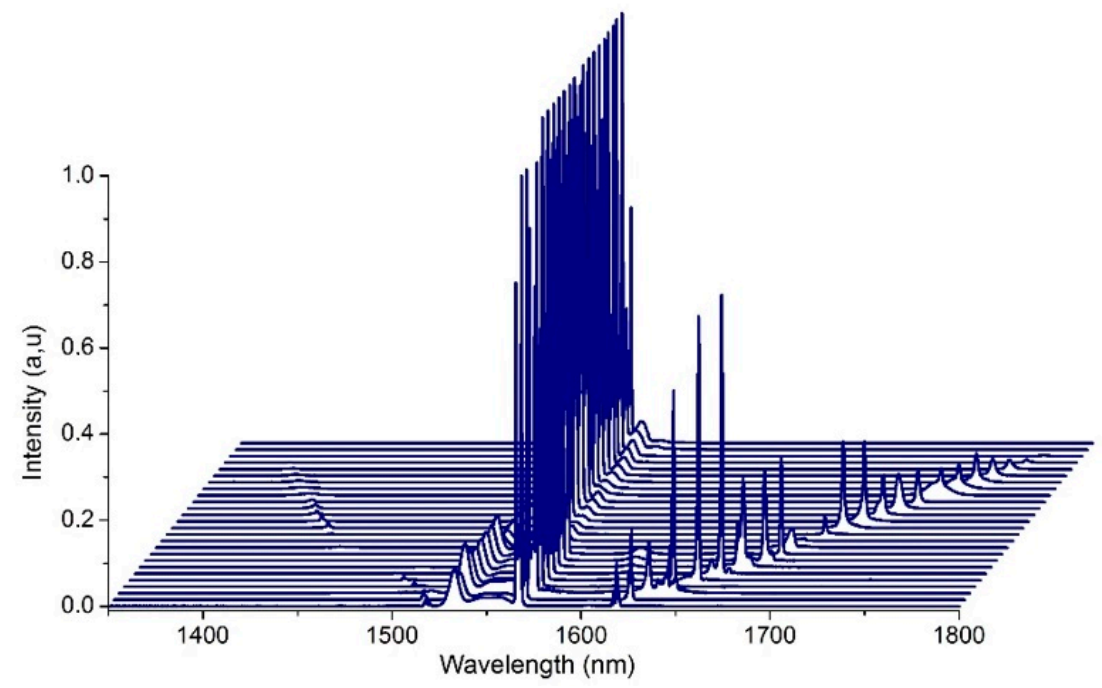

Figure 13. Idler tenability, in linear scale, while tuning the pump wavelength and the optical delay line. 


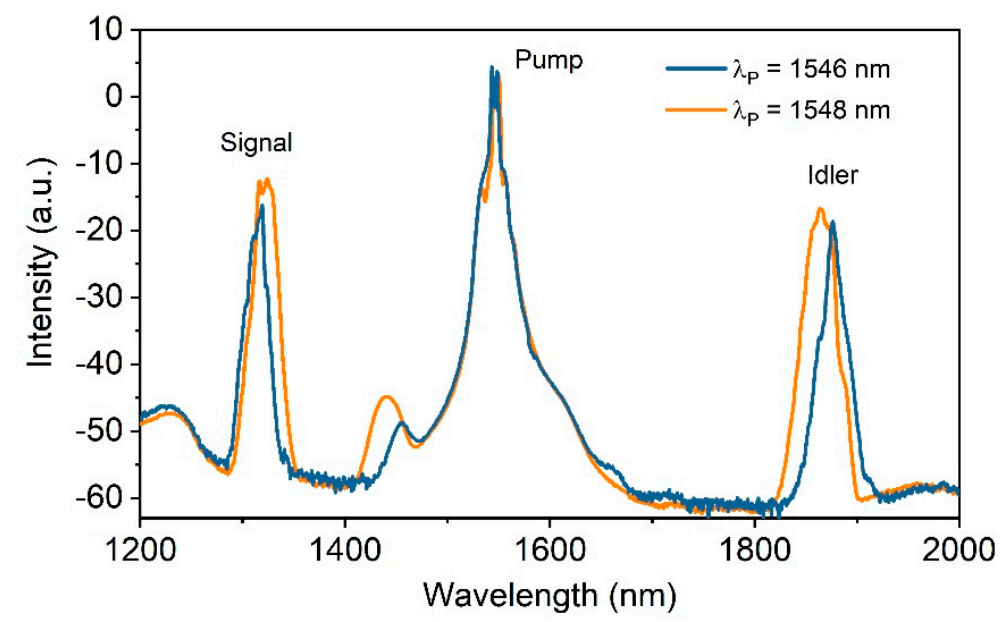

Figure 14. Generation of signal and idler wavelengths close to $1300 \mathrm{~nm}$ and $1900 \mathrm{~nm}$ respectively.

\subsection{Noise Characterizations}

As previously discussed, applications, e.g., in biophotonics and sensing, require highly stable and low-noise pulsed sources. We therefore performed relative intensity noise (RIN) measurements of both the pump dissipative soliton erbium doped fiber laser (DS-EDFL) and the FOPO. The measurement system essentially consists in an InGaAs PIN photodetector (DET08CFC) with a response $r(\lambda)=0.9$ around $1550 \mathrm{~nm}$, an amplifier (MITEQ AU-1442) with a gain of $34 \mathrm{~dB}$, and a noise figure of $1 \mathrm{~dB}$, and an electric spectrum analyzer (Rohde\&Schwarz FSV $7 \mathrm{GHz}$ ). As the laser delivers high power, and does not have to operate near the threshold either (to avoid additional quantum noise), measurements were performed at the $10 \%$ output of coupler $\mathrm{C} 1$ using an optical attenuator, while blocking the FOPO feedback branch. Signals were then measured with a power meter and/or sent to the RIN measurement system. Concerning the FOPO characterization, the filtered idler from the $90 \%$ output of coupler C2 was sent for RIN measurements in the same way. The attenuator was used to control the power that arrived on the detector (DET08CFC) in order to characterize its optical response. From preliminary measurements, we confirmed that the photodetector guaranteed a linear response for incident optical powers up to $1.5 \mathrm{~mW}$.

The performed RIN measurements using this system are shown in Figure 15a. Our pump laser exhibited a good RIN of $-154 \mathrm{dBc} / \mathrm{Hz}$ at $\lambda \mathrm{p}=1566 \mathrm{~nm}$, comparable to the system used in Ref. [6]. The FOPO increased it to around $-140 \mathrm{dBc} / \mathrm{Hz}$, considering only the filtered idler signal in the $-3 \mathrm{~dB}$-bandwidth between $1655 \mathrm{~nm}$ and $1685 \mathrm{~nm}$, which represents a satisfying performance in the frame bio-imaging applications. More investigations are however now needed to further enhance the RIN of the whole system beyond this value.
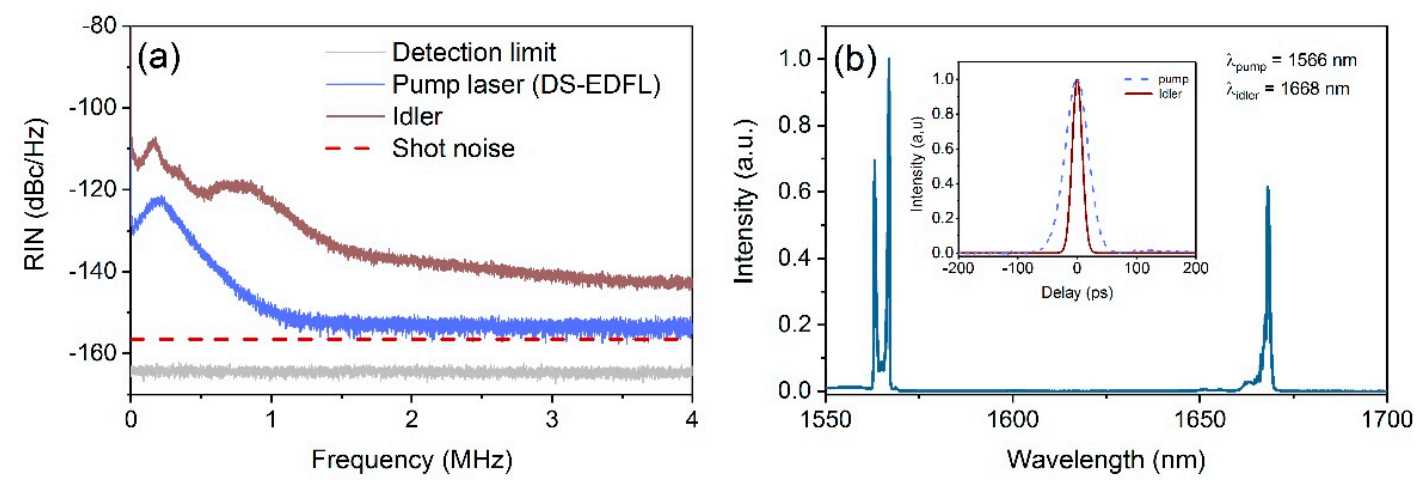

Figure 15. (a) Measured RIN of both pump and idler pulses. These measurements correspond to a $1.4 \mathrm{~mA}$ optical current. (b) Pump and idler spectra at $1566 \mathrm{~nm}$ and $1668 \mathrm{~nm}$, respectively. The inset represents the pump and idler autocorrelation traces. 


\section{Conclusions}

In conclusion, we have demonstrated the generation of high-energy, ultra-short pulses from a dissipative soliton mode-locked fiber laser operating at $1560 \mathrm{~nm}$. Chirped picosecond pulses with up to $38 \mathrm{~nJ}$ energy, which can be de-chirped down to $700 \mathrm{fs}$ duration, have been generated in a large normal dispersion laser configuration with an optimized length of dispersion compensating fiber. This versatile source has then been applied, in a configuration enabling highly-chirped pulses, to advantageously pump a fiber OPO in order to generate picosecond pulses at the nanojoule level in the biological wavelengths window around $1.7 \mu \mathrm{m}$. Such a pumping mode indeed allowed a broad tuning range of more than $200 \mathrm{~nm}$ for the idler wave, with a $30 \mathrm{~nm}$ bandwidth around $1670 \mathrm{~nm}$ where pulses reach the nanojoule level. In addition, the good noise properties of both the erbium fiber laser and parametric oscillator with respective relative intensity noises around -154 and $-140 \mathrm{dBc} / \mathrm{Hz}$ confirm the possibility to implement such a FOPO in the frame of bio-photonics applications.

Author Contributions: Conceptualization, T.G. and A.H.; experimental and numerical investigation, R.B., M.T., P.-H.H., A.T., T.G. and A.H.; custom fibers realization, A.K. and A.M.; saturable absorbers, J.-L.O.; writing-review and editing, R.B., M.T., T.G. and A.H.

Funding: This work was supported by the French Agence Nationale de la Recherche (LabEx EMC3 and CEMPI programs, UBRIS2: ANR-13-BS09-0018, RIFT: ANR-15-CE08-0018-01, Equipex FLUX: ANR-11-EQPX-0017), the European Union with the European Regional Development Fund, and the Regional Council of Normandie.

Acknowledgments: The authors are grateful to A. Le Rouge and L. Bigot for the fabrication of the MCVD preform.

Conflicts of Interest: The authors declare no conflict of interest.

\section{References}

1. Jauregui, C.; Limpert, J.; Tünnermann, A. High-power fibre lasers. Nat. Photonics 2013, 7, 861-867. [CrossRef]

2. Baumgartl, M.; Ortaç, B.; Lecaplain, C.; Hideur, A.; Limpert, J.; Tünnermann, A. Sub-80 fs dissipative soliton large-mode-area fiber laser. Opt. Lett. 2010, 35, 2311-2313. [CrossRef] [PubMed]

3. Lefrançois, S.; Kieu, K.; Deng, Y.; Kafka, J.D.; Wise, F.W. Scaling of dissipative soliton fiber lasers to megawatt peak powers by use of large-area photonic crystal fiber. Opt. Lett. 2010, 35, 1569-1571. [CrossRef] [PubMed]

4. Baumgartl, M.; Lecaplain, C.; Hideur, A.; Limpert, J.; Tünnermann, A. 66 W average power from a microjoule-class sub-100 fs large-pitch fiber oscillator. Opt. Lett. 2012, 37, 1640-1642. [CrossRef] [PubMed]

5. Gottschall, T.; Meyer, T.; Schmitt, M.; Popp, J.; Limpert, J.; Tünnermann, A. Four-wave-mixing-based optical parametric oscillator delivering energetic, tunable, chirped femtosecond pulses for non-linear biomedical applications. Opt. Express 2015, 18, 23968-23977. [CrossRef] [PubMed]

6. Lamb, E.S.; Lefrancois, S.; Ji, M.; Wadsworth, W.J.; Xie, X.S.; Wise, F.W. Fiber optical parametric oscillator for coherent anti-Stokes Raman scattering microscopy. Opt. Lett. 2013, 38, 4154-4157. [CrossRef] [PubMed]

7. Nakazaki, Y.; Yamashita, S. Fast and wide tuning range wavelength-swept fiber laser based on dispersion tuning and its application to dynamic FBG sensing. Opt. Express 2009, 17, 8310-8318. [CrossRef] [PubMed]

8. Wong, G.K.L.; Murdoch, S.G.; Leonhardt, R.; Harvey, J.D.; Marie, V. High-conversion-efficiency widely-tunable all-fiber optical parametric oscillator. Opt. Express 2007, 15, 29472952. [CrossRef]

9. Brinkmann, M.; Janfrüchte, S.; Hellwig, T.; Dobner, S.; Fallnich, C. Electronically and rapidly tunable fiber-integrable optical parametric oscillator for nonlinear microscopy. Opt. Lett. 2016, 41, 2193-2196. [CrossRef] [PubMed]

10. Horton, N.G.; Wang, K.; Kobat, D.; Clark, C.G.; Wise, F.W.; Schaffer, C.B.; Xu, C. In vivo three-photon microscopy of subcortical structures within an intact mouse brain. Nat. Photonics 2013, 7, 205-209. [CrossRef] [PubMed]

11. Nguyen, T.N.; Kieu, K.; Maslov, A.V.; Miyawaki, M.; Peyghambarian, N. Normal dispersion femtosecond fiber optical parametric oscillator. Opt. Lett. 2013, 38, 3616-3619. [CrossRef] [PubMed]

12. Zhou, Y.; Cheung, K.K.Y.; Yang, S.; Chui, P.C.; Wong, K.K.Y. Widely tunable picosecond optical parametric oscillator using highly nonlinear fiber. Opt. Lett. 2009, 34, 989-991. [CrossRef] [PubMed]

13. Saito, S.; Kishi, M.; Tsuchiya, M. Dispersion-flattened-fibre optical parametric oscillator for wideband wavelength-tunable ps pulse generation. Electron. Lett. 2003, 39, 86-88. [CrossRef] 
14. Becheker, R.; Tang, M.; Hanzard, P.H.; Tyazhev, A.; Mussot, A.; Kudlinski, A.; Kellou, A.; Oudar, J.L.; Godin, T.; Hideur, A. High-energy dissipative soliton-driven fiber optical parametric oscillator emitting at $1.7 \mu \mathrm{m}$. Laser Phys. Lett. 2018, 15, 1-5. [CrossRef]

15. Noronen, T.; Okhotnikov, O.; Gumenyuk, R. Electronically tunable thulium-holmium mode-locked fiber laser for the 1700-1800 nm wavelength band. Opt. Express 2016, 24, 14703-14708. [CrossRef] [PubMed]

16. Noronen, T.; Firstov, S.; Dianov, E.; Okhotnikov, O.G. $1700 \mathrm{~nm}$ dispersion managed mode-locked bismuth fiber laser. Sci. Rep. 2016, 6, 24876. [CrossRef] [PubMed]

17. Cadroas, P.; Abdeladim, L.; Kotov, L.; Likhachev, M.; Lipatov, D.; Gaponov, D.; Hideur, A.; Tang, M.; Livet, J.; Supatto, W.; et al. All-fiber femtosecond laser providing $9 \mathrm{~nJ}, 50 \mathrm{MHz}$ pulses at $1650 \mathrm{~nm}$ for three-photon microscopy. J. Opt. 2017, 19, 065506. [CrossRef]

18. Gordon, J. Theory of the soliton self-frequency shift. Opt. Lett. 1986, 11, 662-664. [CrossRef] [PubMed]

19. Mitschke, F.; Mollenauer, L. Discovery of the soliton self-frequency shift. Opt. Lett. 1986, 11, 659-661. [CrossRef] [PubMed]

20. Zhang, L.; Tuan, T.H.; Kawamura, H.; Suzuki, T.; Ohishi, Y. Optical parametric oscillator based on degenerate four-wave mixing in suspended core tellurite microstructured optical fiber. Opt. Express 2015, 23, 26299-26304. [CrossRef] [PubMed]

21. Nguyen, T.N.; Kieu, K.; Gowda, R.; Ota, T.; Uno, S.; Peyghambarian, N. Widely tunable normal dispersion fiber optical parametric oscillator. In Proceedings of the 2014 Conference on Lasers and Electro-Optics (CLEO)—Laser Science to Photonic Applications, San Jose, CA, USA, 8-13 June 2014; pp. 1-2.

22. Grelu, P.; Akhmediev, N. Dissipative solitons for mode-locked lasers. Nat. Photonics 2012, 6, 84-92. [CrossRef]

23. Chong, A.; Renninger, W.H.; Wise, F.W. All-normal-dispersion femtosecond fiber laser with pulse energy above 20 nJ. Opt. Lett. 2007, 32, 2408-2410. [CrossRef] [PubMed]

24. Cabasse, A.; Ortaç, B.; Martel, G.; Hideur, A.; Limpert, J. Dissipative solitons in a passively mode-locked Er-doped fiber with strong normal dispersion. Opt. Express 2008, 16, 19322-19329. [CrossRef] [PubMed]

25. Cabasse, A.; Gaponov, D.; Ndao, K.; Khadour, A.; Oudar, J.L.; Martel, G. 130 mW average power, 4.6 nJ pulse energy, 10.2 ps pulse duration from an $\mathrm{Er}^{3+}$ fiber oscillator passively mode locked by a resonant saturable absorber mirror. Opt. Lett. 2011, 36, 2620-2622. [CrossRef] [PubMed]

26. Chong, A.; Buckley, J.; Renninger, W.; Wise, F. All-normal-dispersion femtosecond fiber laser. Opt. Express 2006, 14, 10095-10100. [CrossRef] [PubMed]

27. Chichkov, N.B.; Hausmann, K.; Wandt, D.; Morgner, U.; Neumann, J.; Kracht, D. High-power dissipative solitons from an all-normal dispersion erbium fiber oscillator. Opt. Lett. 2010, 35, 2807-2809. [CrossRef] [PubMed]

28. Liu, H.; Liu, Z.; Lamb, E.S.; Wise, F. Self-similar erbium-doped fiber laser with large normal dispersion. Opt. Lett. 2014, 39, 1019-1021. [CrossRef] [PubMed]

29. Tang, M.; Wang, H.; Becheker, R.; Oudar, J.L.; Gaponov, D.; Godin, T.; Hideur, A. High-energy dissipative solitons generation from a large normal dispersion Er-fiber laser. Opt. Lett. 2015, 40, 1414-1417. [CrossRef] [PubMed]

30. Schreiber, T.; Ortaç, B.; Limpert, J.; Tünnermann, A. On the study of pulse evolution in ultra-short pulse mode-locked fiber lasers by numerical simulations. Opt. Express 2007, 15, 8252-8262. [CrossRef] [PubMed]

31. Wang, H.; Kotov, L.V.; Gaponov, D.; Cabasse, A.; Yashkov, M.V.; Lipatov, D.S.; Lichachev, M.; Oudar, J.L.; Martel, G.; Février, S.; et al. Dissipative soliton generation and amplification in erbium-doped fibers operating at $1.55 \mu \mathrm{m}$. IEEE J. Sel. Top. Quantum Electron. 2014, 20, 283-289. [CrossRef]

32. Ruehl, A.; Prochnow, O.; Wandt, D.; Kracht, D.; Burgoyne, B.; Godbout, N.; Lacroix, S. Dynamics of parabolic pulses in an ultrafast fiber laser. Opt. Lett. 2006, 31, 2734-2736. [CrossRef] [PubMed]

33. Dinda, P.T.; Seve, E.; Millot, G.; Sylvestre, T.; Maillotte, H.; Lantz, E. Raman-assisted three-wave mixing of non-phase-matched waves in optical fibres: Application to wide-range frequency conversion. Opt. Commun. 2001, 192, 107-121. [CrossRef]

(c) 2018 by the authors. Licensee MDPI, Basel, Switzerland. This article is an open access article distributed under the terms and conditions of the Creative Commons Attribution (CC BY) license (http:/ / creativecommons.org/licenses/by/4.0/). 